\title{
L'implication corporelle dans les situations éducatives
}

La prise en compte du corps de l'enseignant dans les processus didactiques et dans la formation

\section{Gérard Saez}

\section{(2) OpenEdition}

\section{Journals}

Édition électronique

URL : http://journals.openedition.org/trema/620

DOI : 10.4000/trema.620

ISSN : 2107-0997

Éditeur

Faculté d'Éducation de l'université de Montpellier

Édition imprimée

Date de publication : 1 mai 2004

Pagination : 71-83

ISSN : 1167-315X

Référence électronique

Gérard Saez, "L'implication corporelle dans les situations éducatives », Tréma [En ligne], 23 | 2004, mis en ligne le 08 septembre 2010, consulté le 19 avril 2019. URL : http://journals.openedition.org/ trema/620 ; DOI : 10.4000/trema.620

Ce document a été généré automatiquement le 19 avril 2019

Trema 


\section{L'implication corporelle dans les situations éducatives}

La prise en compte du corps de l'enseignant dans les processus didactiques et dans la formation

\section{Gérard Saez}

1 Il est des questions délicates, voire agaçantes, qu'on ne peut pour autant évacuer.

2 En voici quelques-unes qui ont présidé aux recherches initiales :

- Dans le cadre du module de formation des futurs enseignants intitulé « Le Corps et La Voix », peut-on proposer des modèles corporels identifiables,

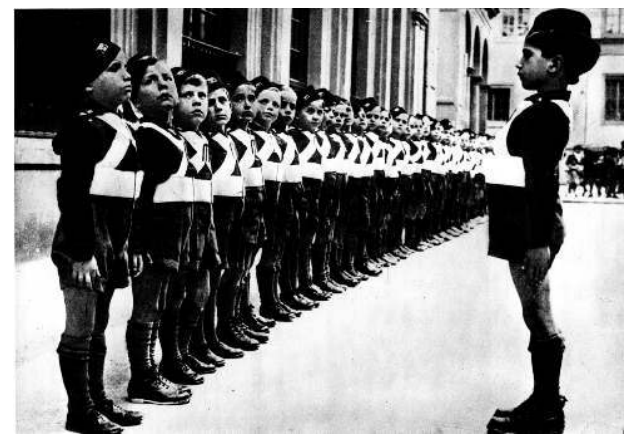
ou tout au moins des systèmes de référence?

- Quelles sont les représentations véhiculées par les individus et les groupes, et que signifient-elles, que valent-elles?

- Finalement, où est le corps de l'enseignant ? Doit-on et peut-on même entreprendre un travail de formation à ce niveau ? Ou ne serait-il pas plus raisonnable de s'en tenir à une information?

3 La présente contribution ${ }^{1}$ se veut le reflet de ce questionnement, non encore totalement abouti, et s'appuie sur la pratique menée au sein de l'IUFM de Montpellier.

4 J'entamerai donc mon propos en évoquant les prémices de certaines séances de formation des futurs PE et PLC, principalement les stagiaires de deuxième année. 


\section{L'image de l'enseignant}

5 Le travail commence par une mise à plat des représentations mentales de ce qu'est un enseignant. Les étudiants et stagiaires sont invités à dire 'à quoi ressemble un enseignant' selon eux. Cette verbalisation servira de base à un travail d'autoscopie qui permettra d'explorer l'image de soi et le rapport entretenu avec elle. Pour lancer le processus, je leur fournis un corpus iconographique, constitué pour l'essentiel à partir des ressources de l'IUFM. Par souci d'objectivité, le dit corpus n' a pas été établi par moi seul: j'ai demandé à des collègues et à des étudiants de me fournir des « images d'enseignants en train d'enseigner"; j'ai ainsi obtenu un recueil (évolutif) d'une cinquantaine de documents divers, balayant diachroniquement et synchroniquement le thème. Je demande aux participants de repérer dans chaque image l'enseignant et de formaliser les critères d'identification. Certains éléments socio-historiques peuvent ainsi être abordés et on vérifie aisément que l'iconographie véhicule les valeurs plus ou moins conscientes des époques et des milieux. A titre d'exemple, deux gravures jumelles du XVIIe siècle intitulées respectivement le maitre d'école et la maîtresse d'école montrent que le concept d'enseignement pour les filles est alors fort mal défini : si le «maître d'école » est parfaitement repérable grâce à des marqueurs que les stagiaires peuvent formuler, tels que l'âge ou d'autres signes de "maturité » (barbe, lunettes,...), la supériorité spatiale (fonctionnelle et symbolique), la situation de confrontation, l'espace privilégié et protégé, les attributs (tenue stricte et / ou codifiée, couvre-chef parfois, livre ou autre support du savoir, règle / bâton et / ou autres instruments de démonstration et / ou coercition, ...), la "maitresse d'école » appelle toujours à discussion et, au final, on ne peut trancher catégoriquement. Cette représentation iconographique «floue» me semble être la manifestation d'une représentation mentale tout aussi problématique : à l'époque de Molière, l'éducation féminine n'a pas de statut social « sérieux ».

6 Mais ce qui, au bout d'un certain temps, émerge de l'observation de ce corpus, c'est la récurrence quasi systématique (au point qu'elle n'est d'abord pas repérée comme signifiante ...) d'une attitude, ou plutôt d'un geste qui apparaît ainsi comme la marque première de l'enseignant : il s'agit du geste de démonstration, bras tendu et index pointé ${ }^{2}$. La règle (ou le bâton), instrument polyvalent (tracer, montrer, punir), en est le prolongement naturel, mais le geste de l'index seul, statistiquement plus fréquent, semble encore plus chargé de symbolique.

7 Ce geste ne peut manquer d'évoquer un autre univers iconographique où il figure largement: celui des représentations religieuses (et point n'est ici besoin de rappeler l'étroit lien historique qui unit les enseignants et les « clercs»). C'est le geste par lequel, au plafond de la Chapelle Sixtine, le Créateur insuffle la vie à sa créature, c'est celui de Sainte ANNE, de la Vierge MARIE ou de l'enfant JESUS qui, dans les Maternités, permet à celui ou celle qui sait d'indiquer à l'autre les paroles des Ecritures, c'est peut-être le geste de bénédiction du Pancratos. Geste de pouvoir, de connaissance et de transmission, on le retrouve dans la « main de justice » du souverain ou dans le doigt pointé du jeteur de sort (dont la crainte survit dans l'interdiction faite aux enfants de "montrer du doigt ») ... et dans l'index d'E.T.!

8 A vrai dire, il n'est pas étonnant que l'enseignant soit « celui qui montre ", puisque c'est ce que révèle l'étymologie du terme: *in-signare, depuis le XIe siècle, c'est bien «faire 
signe », et même "faire signe vers». Suffirait-il donc, pour devenir un «bon» enseignant, d'apprendre à pointer l'index avec quelque élégance?

\section{Un corps transparent}

«Le sage montre la lune, et le fou regarde le doigt». Cet aphorisme chinois dépeint une situation d'enseignement pervertie. L'apprenant doit normalement s'affranchir de la personne de l'enseignant. Il lui faut l'abandonner pour accéder aux objets de connaissance, extérieurs à la relation duelle maître / élève et visés par elle. Le corps de l'enseignant est un lieu de passage vers les savoirs. Il ne doit en aucun cas devenir un obstacle car il doit être lu comme intention et non comme support. Transitoire, il doit tendre à la neutralité et sa matérialité immédiate est à proprement parler insignifiante. Un corps qui fait signe et non un corps qui est signe. Suivant le bras, la main puis le dactyle final, le regard de l'élève s'affine, s'éthérise pour s'envoler vers les concepts, et simultanément la personne de l'enseignant devient comme virtuelle, évanescente, permettant d'autant mieux une sorte de mimésis (au sens girardien du terme) cognitive, non conflictuelle parce que transférée, désincarnée.

Et de fait dans l'imagerie idéale, comme l'homme était censé disparaître sous la soutane du prêtre, l'individu enseignant s'est longtemps dissimulé sous la robe, l'uniforme ou la blouse de sa fonction. Cette vêture sobre s'assortit naturellement d'une allure générale de modération et de dignité et d'un comportement «équilibré ». Le principe se décline différemment selon les époques et les contextes, mais il perdure en leitmotiv dans les consignes et recommandations officielles et professionnelles, dans la littérature et dans l'imagerie collective et, nous le verrons, s'il peut sembler aujourd'hui remis en cause, c'est justement parce que certains faits sont perçus comme dérogeant à cette norme implicite. Bien sûr, l'uniforme noir des hussards de la république ou la blouse grise de l'instituteur ne sont plus vraiment de mode, mais la sobriété vestimentaire est encore souvent de mise et j'ai entendu plusieurs fois des stagiaires et des enseignants, débutants ou non, déclarer qu'ils s'habillaient de façon plus stricte pour «mieux » exercer leur tâche. Cette neutralité recherchée éliminerait les parasites et les bruits dans une relation didactique ritualisée, dépouillée des interférences personnelles. Il est bien connu que "l'enseignant doit laisser ses problèmes privés » au vestiaire, on peut même y laisser toute son affectivité, sauf si elle entre partiellement dans le cadre professionnel comme en Maternelle et, déjà dans une moindre mesure, dans l'élémentaire.

11 Mais comment un individu en situation sociale pourrait-il « ne pas communiquer »? En s'effaçant derrière un réseau de signes plus forts qui affirment que le personnage social (incarnation des valeurs collectives) se construit par évacuation de la corporalité individuelle (victoire du Surmoi sur le Ça en quelque sorte).

\section{L'évacuation du corps}

12 La place du corps dans les processus cognitifs est encore l'objet de discussions. P. ARNAUD (1983) montre brillamment comment les recherches, de PIAGET et WALLON à LAPIERRE en passant par Le BOULCH, ont fait évoluer la façon dont ce rapport était appréhendé. Le «mens sana in corpore sano » de JUVENAL a maintes fois été proclamé mais, outre qu'il peut s'interpréter de diverses manières, sa mise en pratique apparaît 
comme plus problématique, et l'on sait que les recommandations les plus répétées le sont souvent parce qu'elles ne sont pas les plus suivies.

Le Fascicule de Documentation Administrative de 1961, Les Elèves-Maîtres, stipule que pour entrer dans la carrière, il faut être exempt de maladies telles que la tuberculose et autres « affections contagieuses et transmissibles » ou encore de diverses affections organiques telles que les troubles cardio-vasculaires, la leucémie, la cirrhose, le diabète ou les dystrophies d'origine endocrinienne, sont également motifs d'exclusion les "affections du système nerveux » dont l'épilepsie, les tics convulsifs et les intoxications chroniques mais aussi les difformités et cicatrices par trop disgracieuses. Cette préoccupation est sans doute ici double, le corps doit être un instrument fonctionnant correctement ${ }^{3}$ et il doit offrir une image lisse et confortable.

Mais quand ALAIN (1926), affirme que «le corps humain est le tombeau des Dieux» dont il faut dompter le "troupeau de muscles», et quand P. BOURDIEU (1979), décrit le plaisir intellectuel et esthétique des individus socio-culturellement dominants comme trouvant sa source dans le détachement de la « nécessité physiologique » et de la « réalité en chair et en os ", le corps se trouve renvoyé dans la dichotomie manichéenne qui faisait de la matière l'œuvre Mauvaise dont l'esprit devait se libérer. Ou, tout au moins, il est relégué au fond de la caverne platonicienne, abusé par les illusions de ses sens grossiers.

Le corps est alors le lieu de l'animalité, des pulsions, de l'irrationnel. Il faut l'apprivoiser ou, mieux, le domestiquer pour que l'esprit s'épanouisse. Cette conception ne peut être considérée comme « dépassée » et hors de propos : elle est encore bien présente dans les imaginaires collectifs ${ }^{4}$ et individuels et, dans le cadre professionnel qui est le mien, je me dois de la prendre en compte. J'essaierai de montrer plus loin quelle importance il convient de lui accorder.

De même, j'essaierai d'expliquer pourquoi je pense que le corps enseignant (ici au sens de corporation) est fondamentalement partagé entre un "progressisme intellectuel» d'impulseur de la connaissance et un "passéisme intellectuel» de conservateur des savoirs. Et dans ces savoirs j'inclus les concepts, symboles et valeurs de l'imaginaire collectif, dont la vision du corps (ici individuel) évoquée plus haut.

Bien que celle-ci soit déjà ancienne, on peut donc encore trouver des échos à la pensée d'ALAIN (op. cit.) qui, écrivant que « la civilisation se reconnaît à ce calme du visage et du corps qui s'oppose si bien à l'agitation des enfants ", expulse de la faculté d'abstraction rationnelle les incohérences et les trivialités qui agitent l'affectif et le physiologique.

\section{Conflits}

Si j'utilise depuis le début de mon propos le mot « enseignant » au masculin, c'est bien sûr comme terme générique et si la plupart des figures évoquées sont masculines, ce n'est pas que j'ignore que la profession s'est grandement féminisée au cours du siècle écoulé, mais c'est parce que l'entité "enseignant» est jusqu'ici considérée comme asexuée (la sexualité étant évacuée comme relevant de la corporalité animale) ou, dans certains contextes socio-historiques, d'une virilité «allant de soi», «normale», et nullement dialectique.

19 Je continuerai, sauf nécessité particulière, à user de ce terme masculin générique (l'option "par défaut») par commodité car prendre en compte les particularités de chaque sexe, tant pour les élèves que pour les enseignants, nous entraînerait trop loin et hors du cadre 
de cet article. On voudra donc bien retenir que certains des éléments énoncés peuvent se décliner avec des variations notables selon le sexe des protagonistes. Par exemple, les comportements de violence physique qui seront évoqués sont plus spécifiques des élèves masculins mais, outre qu'ils ne le sont pas exclusivement, il est certains comportements féminins spécifiques qui peuvent fonctionner comme équivalents. Violence. Le mot est lâché et aussitôt les images de l'actualité affluent. Violence, agressivité, vandalisme, provocations, irrespect et ignorance dont se plaignent certains enseignants, apparemment de plus en plus nombreux, et dont journaux et télévisions font fréquemment leurs titres. Le corps de l'enseignant est arraché à son estrade éthérée et plongé tête première dans ces classes chaotiques où des garnements avachis ou injurieux flanquent des coups de pied aux savoirs, aux institutions et éventuellement à ceux qui les professent. Pourquoi, quand la situation didactique dérape, la violence physique, métaphorisée ou effective, surgit-elle si facilement? C'est qu'effectivement, comme le proférait ALAIN, le corps peut être un ennemi redoutable pour l'esprit.

L'activité physique peut être envisagée comme dérivatif ou «antidote» au travail de l'esprit (dans ses dimensions intellectuelle et morale), conception qui prévalait par exemple lorsque l'Education Physique servait à lutter contre le "surmenage scolaire», ou, si elle est correctement canalisée, comme support à celui-ci, mais la corporalité peut aussi devenir une négation de l'activité mentale.

Si les clercs claironnent que « la plume est plus forte que l'épée», cela signifie aussi que toutes deux sont fondamentalement en conflit. La violence physique et l'usage de la force, l'histoire l'a assez montré, font bon ménage avec l'obscurantisme et la sclérose intellectuelle. Si l'homo sapiens a prospéré grâce à son cerveau plus qu'à ses muscles ${ }^{5}$, le jeu social rappelle aisément que la puissance physique n'est pas un facteur négligeable à l'échelle inter-individuelle. Cogner pour faire taire l'importun est un « je ne veux pas le savoir» efficace et ${ }^{2}$

Dans son ouvrage passionnant, S. BOIMARE (1999) explore le mythe d'Héraclès et explique comment il en fait un outil pédagogique révélateur des inhibitions psycho-cognitives menant à un surinvestissement corporel violent. HERACLES, empêtré dans les conflits (son nom d'ALCIDE ainsi que nombre de ceux des membres de sa famille évoque la lutte, le combat), les faux-semblants (les thèmes de la dualité et de la gémellité chers à GIRARD parsèment le récit), les incertitudes et les non-dits, choisit d'étouffer les voix du savoir et se construit un rempart de muscles dont l'impénétrabilité sera confortée ultérieurement par la peau du Lion de NEMEE. Hostile à toute connaissance, il est l'élève abominable par excellence puisqu'il est un tueur d'enseignants (son maitre de musique Linos puis, quoique accidentellement, le centaure Chiron, archétype des mentors antiques).

Ce corps-cuirasse est d'une virilité exacerbée et la violence est posée comme valeur mâle, brutale, opposée à l'intellectualité inconsistante, insidieuse, féminine. On trouve ici un écho de ce que D. ANZIEU (1995) appelle, à la suite d'E. BICK, la " seconde peau musculaire», enveloppe protectrice d'un psychisme par trop conflictuel. Cette enveloppe somatique de muscles et de violence contient les dissensions internes et s'oppose aux stimulations externes gênantes mais elle est aussi disponible comme moyen d'action sur l'environnement.

25 La violence scolaire subie par l'enseignant ${ }^{6}$ l'empêche tout simplement d'enseigner, elle évite donc aux élèves d'apprendre et leur démontre même que la " domination» physique vaut bien la "supériorité» intellectuelle. Car plus moyen alors d'espérer que le 
regard de l'apprenant glissera avec délicatesse sur le corps sublimé de son maitre pour aller contempler les beautés abstraites de la connaissance. Le corps est bien là, un corps fragile d'individu qui ne peut s'empêcher de " communiquer» autre chose que le seul contenu de ses discours didactiques, qui peut cristalliser des conflits personnels, sur lequel on peut avoir prise. Les divers masques idéologiques qui ont pu parfois protéger les enseignants de chair et de sang ont perdu de leur consistance. Et d'aucuns en viennent à regretter les estrades, les distributions de prix, voire les blouses et les plumes sergentmajor, tout l'apparat rituel qui auréolait, à l'époque bénie qu'on ne précise pas, l'Enseignant porteur des Vraies Valeurs.

Il n'est pas opportun d'épiloguer ici sur les origines de la « crise de l'école» ni sur la « fracture sociale», ni même sur le problème sur le problème de "l'autorité» ou des " valeurs» mais force est de constater que l'aspiration idéale vers les savoirs ne semble plus très opérante. Et c'est l'enseignant in corpore qui se trouve en première ligne pour affronter l'onde de choc, mis bien malgré lui peut-être... au centre du système éducatif (oserai-je dire « à l'épicentre»?). Cet être qui se concevait transparent devient lourd et opaque. Ne pouvant plus être évacués vers les objets de connaissance ${ }^{7}$, les conflits se déversent sur sa tête: incarnation entre autres d'un Savoir rejeté (quand on n'aime pas les maths, on n'aime pas le prof de maths), d'une Ecole dépréciée (vécue par certains comme le lieu de l'échec et de la frustration), d'une Société reniée (l'exclusion est un phénomène facilement réciproque) et éventuellement en prime objet de transfert des tensions familiales, l'enseignant doit assumer tout cela en sus de sa gestion de la relation inter-individuelle.

Apparaît alors une alternative qui me semble fort bien illustrée par le témoignage diversement apprécié de M. GOYET (2003) ${ }^{8}$

Jeune « prof de banlieue» confrontée à une situation scolaire dépeinte comme déliquescente, l'auteur stigmatise la dérive vers le prof « un peu mono, un peu G.O., un peu assistant social, très pote et complice, pas mal aîné-grand frère», bref vers le choix de la relation inter-individuelle " horizontale» sauvée par l'abandon de la relation « verticale» aux Idéaux.

Ce premier choix se traduit notamment par l'amollissement, le relâchement et la pesanteur (de la déléguée syndicale caricaturée, on retient surtout-est-ce un hasard ?-son " énorme masse»), l'enfoncement dans la matière et la vulgarité.

L'autre choix est celui de la « droiture», de la Loi («travailler, transmettre, exiger»), bref de la Règle.

\section{Le problème de la norme}

31 Lorsque sont fondés en France les premiers établissements spécifiquement dévolus à la formation des enseignants, ils prennent le nom d'Ecoles Normales. L'institution combine un idéal de laïcité républicaine et un héritage clérical et militaire. Dans ce contexte sociohistorique, «norme» est un concept étroit, rigide, essentiellement vertical et laissant peu de place aux spécificités. L'enseignement a peu de scrupules à être directif et autoritaire sur le plan moral, intellectuel et corporel. Le corps de l'élève est ainsi l'objet de directives strictes, la vie de classe exclut tout débordement physiologique, du bavardage à l'agitation, et prescrit des attitudes corporelles culturellement codifiées. Les pratiques physiques sont liées aux contextes socio-historiques (de la préparation militaire des 
premières décennies aux pratiques «naturelles») et, on l'a dit, elles évolueront au rythme de la réflexion scientifique et pédagogique (cf. P. ARNAUD Les savoirs du corps op. cit. et aussi Le militaire, l'écolier, le gymnaste). Le concept de " norme» évoluera lui aussi sémantiquement pour des raisons diverses et laissera plus de place aux spécificités, on passera d'une norme-contrainte à une norme-référence (cf. G. CANGUILHEM (1966). sur la moralité (ce qui inclut le « comportement», mais comme habitus à valeur sociale et non comme phénomène psycho-physiologique) et les compétences et pratiques intellectuelles, le corps stricto sensu ne fait pas l'objet de références spécifiques (hormis, on l'a dit, une «normalité» physiologique fonctionnelle). Les pratiques physiques dans les Ecoles Normales, par exemple, sont le calque des pratiques scolaires et, pour l'essentiel, les Elèves-Maîtres apprennent à «faire faire». Les autres dimensions de la corporalité n'arriveront que très tard dans le cursus de formation des enseignants et surtout elles restent longtemps purement théoriques. Après PIAGET et WALLON, bien sûr, on ne pouvait plus ne pas faire de la psychopédagogie et ne pas s'intéresser à la psychomotricité mais là encore il s'agissait de «comprendre» le corps de l'autre (de l'élève) pour agir sur lui, et non d'œuvrer sur son corps propre (celui de l'enseignant). Le travail mené depuis peu dans les modules «Corps et Voix» des IUFM et l'évolution remarquable des STAPS essayent de répondre à cette problématique. Mais ce processus est récent et encore mal installé dans les pratiques et dans les mœurs. C'est que le concept de Norme comme cadre absolu et exclusif n'est pas à jeter aux oubliettes. Beaucoup d'enseignants sont fondamentalement hostiles à toute idée de travail sur leur corporalité propre, vécu comme une intolérable intrusion totalitaire. Il est vrai que l'Histoire a donné des exemples de «formatage» des enseignants qui ne nous semblent plus déontologiquement acceptables. Mais ce refus affiché d'une norme trop contraignante peut empêcher toute réelle prise en compte des normes de référence effectives. Et lorsque les besoins de repères (ou de modèles) se font sentir, comme dans 
les situations éducatives conflictuelles évoquées supra, les archétypes qui ressurgissent sont souvent mal maîtrisés.

Dans le témoignage de M. GOYET (op. cit.), l'évacuation du corps est manifeste. Celui des élèves est évoqué principalement dans un chapitre intitulé «Fonctionnement et Rouages». Cette surprenante étiquette mécaniste (elle est censée faire référence aux mécanismes institutionnels, évoqués dans le chapitre, mais elle " colle» aussi à des thèmes purement corporels) évoque pour moi l'univers du plasticien australien STELARC pour qui le corps est « obsolète» et qui, prolongeant le principe d'extériorisation de $\mathrm{M}$. MC LUHAN (1968), adjoint à son organisme des dispositifs prothétiques sophistiqués. Ce corps rappelle aussi les "animaux machines» de DESCARTES, mais on en souligne l'archaïsme, la grossièreté. Il est essentiellement défectueux, irrationnel et perturbateur. Dans Collèges de France, les comportements des Aides-Educateurs qui s'adonnent au skateboard sont qualifiés « d'Infantilismes» et lorsque les dits individus veulent déstabiliser la jeune enseignante, ils glissent sous la porte de sa classe des "photos pornos». La sexualité, expression primitive de la corporalité, semble d'ailleurs l'objet d'une évacuation systématique. Lorsque M. GOYET est confrontée à un élève masturbateur et qu'un collègue inconscient, maladroit ou ironique fait référence à la tenue vestimentaire de l'enseignante, la dénégation est aussitôt hyperbolique ("Je n'avais pas mis ce jour-là ma tenue panthère!»). On peut d'ailleurs s'interroger sur la part exacte d'ironie de l'auteur dans le chapitre intitulé « Masochisme»: le support pédagogique utilisé (dont la valeur n'est pas ici en question), la projection d'un film muet en noir et blanc sur les Eskimos ${ }^{10}$ s'inscrit fortement dans une perspective d'ascétisme et le titre même de "Masochisme» (puisque recherche d'un conflit perdu d'avance, à la fois souffrance et jouissance) peut faire songer encore une fois à D. ANZIEU (op. cit.) évoquant «l'enveloppe de souffrance» , revanche aussi de l'esprit impuissant à maîtriser le contexte et retournant contre son corps propre la violence qui ne peut être évacuée ailleurs. On retrouve nettement chez M. GOYET les attitudes analysées par P. Bourdieu (op. cit.) et traduisant ce que S. BEAUD (2003) considère comme un « ethnocentrisme de classe» ${ }^{11}$.

\section{Où est le corps de l'enseignant ?}

38 Les enseignants sont à la fois des produits du système éducatif et les producteurs de ce même système. L'axiome sous-jacent est souvent que pour devenir un bon enseignant, il faut et il suffit d'être un bon élève. Mais ce principe est fallacieux. L'écart grandissant entre les normes socio-culturelles des enseignants et de la majorité de leurs élèves désamorce les effets mimétiques escomptés : on ne peut plus raisonnablement espérer que les Normes s'imposeront d'elles-mêmes uniquement, comme semble le croire M. GOYET, parce que l'enseignant les respecte et les incarne. Pire : on peut supposer qu'une bonne partie des enseignants est justement issue du bataillon d'élèves chez qui l'évacuation mimétique du corps a trouvé un écho trop favorable.

Malgré le «mens sana in corpore sano» évoqué et malgré le travail mené par les chercheurs et les praticiens, force est de constater que la population scolaire se répartit encore largement en " intellos " physiquement peu performants et «sportifs» intellectuellement assez ternes. Je crois en effet que ce système de production / reproduction des enseignants occasionne des archaïsmes des représentations non conscientisées et j'en veux pour preuve l'interprétation à mon sens surprenante que fait S. BOIMARE (op. cit.) d'un autre personnage clé du mythe d'Héraclès ${ }^{12}$. S. BOIMARE voit en 
effet dans Eurysthée un pédagogue éclairé qui oblige Héraclès à « un retour en arrière pour approcher et maîtriser ses craintes infantiles qu'il ne sait qu'évacuer». Mais, outre que la traduction du patronyme du personnage me semble abusive ${ }^{13}$, le mythe montre clairement qu'EURYSTHEE n'a rien de bienveillant (il finira d'ailleurs par périr en tentant d'éliminer la descendance d'HERACLES) et les tâches imposées ressemblent plutôt à des tentatives d'élimination de l'encombrant cousin. Eurysthée ne maîtrise nullement la situation et, horrifié, il est incapable d'assumer les produits des quêtes d'HERACLES qui sont refusés, abandonnés ou mis hors-jeu. Par deux fois au moins, (le sanglier d'ERYMANTHE et le chien CERBERE) le monstre rapporté l'effraie tellement qu'il se cache dans une jarre, comme un petit enfant qui fait disparaitre son corps pour faire disparaître le monde. Autre type gémellaire du mythe, cet aîné abusif est un prématuré (HERA a hâté sa naissance pour lui donner la suzeraineté sur HERACLES qui est lui un "surmaturé») qui, à mon sens, illustre le pari opposé à celui d'HERACLES : celui-ci tente d'étouffer le mental sous la carapace corporelle, celui-là essaie de plier le corps sous le joug de l'esprit. Et tous deux échouent. La position de S. BOIMARE me paraît alors fortement éclairante : face à des élèves-HERACLES, on a souvent en effet des enseignants-EURYSTHEE. Les «mauvais élèves» renverraient donc à l'enseignant ce qu'il a refusé d'être.

Cette dénégation / évacuation du corps qui correspond à une conception de l'enseignant plus que jamais inopérante, on en trouve une illustration frappante dans l'histoire de l'enseignement de l'EPS (j'utilise ici la dénomination la plus large). P. ARNAUD (op. cit.) a bien montré comment les enseignants ont toujours manifesté une extrême réticence aux pratiques corporelles. L'enseignement de l'EPS (sous des formes très diverses) prôné dès les premières décennies s'est heurté à un problème majeur : la formation des enseignants avait été totalement négligée et, de surcroît, ceux-ci "oubliaient» rapidement d'appliquer les directives ministérielles. L'enseignement de l'EPS a donc longtemps été coupé des autres pratiques pédagogiques et assuré par des moniteurs plus ou moins qualifiés (à l'origine, des militaires). De nos jours encore, cet état de fait perdure de façon larvée à l'école primaire dans l'utilisation d'intervenants divers qui «déchargent» les enseignants de l'investissement corporel éducatif. Même en Maternelle où la psychomotricité devrait être intégrée aux processus d'apprentissage il est des enseignants qui arguent de leur " incompétence» (le prétexte est aussi vieux que l'Ecole) pour abandonner à d'autres ces activités. Quant au Secondaire et au Supérieur, si on n'est pas enseignant d'EPS ...

41 Dans ma pratique professionnelle, je suis souvent confronté à ces refus et à ces craintes (et même dans les modules de formation "Corps et Voix», on concède plus facilement des heures à la préparation des oraux de concours qu'à l'approche du métier). La remise en question du vécu corporel est pour beaucoup totalement inacceptable. Pourtant, on devrait se rappeler que le rapport au corps est largement construit culturellement et que, si on a appris, on peut continuer à apprendre (surtout pour des enseignants!), ce n'est pas se renier qu'évoluer. Il ne s'agit pas en effet d'inculquer des modèles normatifs prédéfinis, de toute façon, ceux-ci (s'ils existent) ne seraient pas déontologiquement ${ }^{14}$ acceptables: toute technique didactique ou pédagogique dont les résultats seraient parfaitement prévisibles quel que soit l'objet auquel elle s'applique reviendrait à nier ce dernier et serait donc du dressage et non de l'éducation. Il s'agit simplement de prendre en compte les phénomènes avec lucidité. en place des dispositifs réellement impliquants car vouloir encore évacuer vers les seuls 
savoirs les problèmes de la confrontation physique ne pourrait qu'accroître les difficultés. Bien évidemment, les supports théoriques ont toute leur place dans la formation des enseignants. On ne peut ignorer les données de la biologie endocrinienne dans l'approche des manifestations émotionnelles (cf. J. D. VINCENT, 1994), de la sociologie (au moins depuis E. HALL et E. GOFFMAN), de l'éthologie (par exemple B. CYRULNIK), de la sociolinguistique, de l'anthropologie et de la neurophysiologie, entre autres, mais, dans mes modules de formation, je fais aussi place à des pratiques de groupe, à l'autoscopie, à des exercices de prise de parole qui sont des situations réelles.

J'utilise aussi des techniques de jeu dramatique (improvisations individuelles et collectives sous contraintes diverses) et, ce qui peut paraitre plus surprenant, des exercices issus des arts martiaux. Il n'est pas ici possible de développer ce dernier point mais je crois fermement qu'il y a dans ce domaine des ressources de formation très appréciables. L'Aïkido Kobayashi que je pratique et enseigne depuis déjà quelques années permet ainsi d'appréhender les situations conflictuelles (car toute situation d'enseignement est une situation conflictuelle) en cherchant une résolution non-violente au bénéfice des deux participants, sans pour autant nier l'éventuelle violence primordiale. Les rituels conservés permettent aussi une réflexion "vivante» sur les paramètres culturels de la relation éducative. Pour plus d'information, je renvoie au numéro 8 / 9 de la revue universitaire Daruma (2000 / 2001) et au Corps conscient. d'A. COGNARD (2001).

Tout cela me semble plus que jamais nécessaire pour former les enseignants d'aujourd'hui et, pour ce faire, il est indispensable de réaliser que :

- Le corps de l'enseignant ne peut plus être nié.

- La seule modélisation mimétique est de moins en moins opérante, elle est déontologiquement très discutable et elle induit un passéisme néfaste.

- Il est possible de construire des systèmes de référence permettant une gestion plus lucide des situations éducatives.

- L'expérience corporelle ne peut être totalement remplacée par un discours à son propos.

- Le corps de l'enseignant ne peut donc plus être considéré comme un « vieux corps d'élève». 


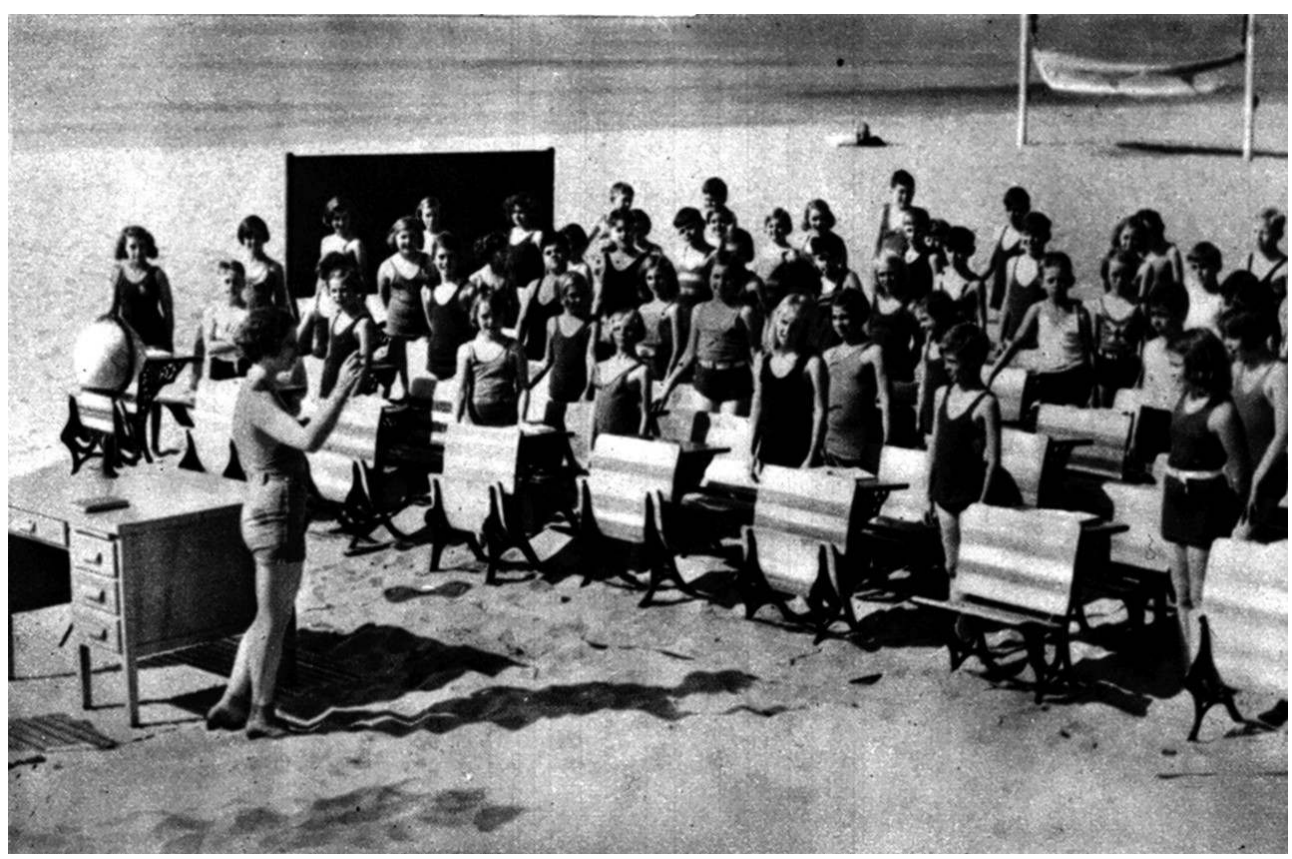

Une expérience : la classe sur la plage, Californie, Etats-Unis

Image (légèrement réduite) tirée de : Enfants à travers le monde / M. Brunot.-Paris : Horizon de France, 1937.-"Le visage de l'enfance".-p. 322

\section{BIBLIOGRAPHIE}

ARNAUD, P. (1983), Les savoirs du corps. Paris : PUL.

ARNAUD, P. (1991), Le militaire, l'écolier, le gymnaste. Lyon : PUL.

ALAIN (1926), Système des Beaux-Arts. Paris : Gallimard.

BOURDIEU, P (1979), La Distinction. Paris : Minuit.

BOIMARE, S (1999), L'enfant et la peur d'apprendre. Paris : Dunod.

ANZIEU, D (1995), Le Moi-peau. Paris : Dunod.

GOYET, M (2003), Collèges de France. Paris : Fayard.

BEAUD, S (2003), in Les Inrockuptibles. numéro du 5 / 11 mars, Paris.

CANGUILHEM, G (1996), Le normal et le pathologique. Paris : PUF.

ZEKRI-HURSTEL, R (2001), Un nouveau regard sur l'élève. Paris : Rouergue.

MC LUHAN, M (1968), Pour comprendre les média. Paris : HMH.

VINCENT, J.D (1994), Biologie des passions. Paris : O.Jacob.

Numéro 8 / 9 de la revue universitaire Daruma, automne 2000 / printemps 2001. Toulouse : P. Picquier.

COGNARD, A (2001), Le Corps conscient. Paris : Dervy. 


\section{NOTES}

1. Rédigée au printemps 2003, elle ne prend pas en compte les événements qui ont secoué depuis le monde enseignant et qui éclairent peut-être sous un jour différent certains des faits abordés 2. Avec généralement, signalons-le, un léger relâchement, l'index dardé ou dressé avec rigidité est plutôt réservé aux gestes de menace ou d'admonition... qui sont d'ailleurs sans doute aussi des formes d'enseignement : ne s'agit-il pas encore de « donner une leçon»?

3. C'est l'esprit par exemple des Instructions d'EPS de 1959 qui conçoivent les pratiques corporelles comme préalable aux activités intellectuelles, une sorte de « maintenance du hardware».

4. Il faudrait considérer aujourd'hui les variations apportées par la pluralité culturelle mais on ne peut aborder ce point ici.

5. Pour ne pas entrer dans les détails, mettons à part ceux de la main ... Je renvoie aux travaux d' A. LEROI-GOURHAN pour plus ample réflexion sur le problème, qui nous ramène d'ailleurs peutêtre au " geste démonstrateur» évoqué au début de cet article.

6. Le problème d'une violence physique, institutionnelle ou non, exercée par l'enseignant sur l'élève ne peut être traité ici.

7. Je ne peux m'empêcher de penser à ce cas d'un professeur du secondaire débordé qui demandait désespérément « quels supports» pourraient lui permettre de relancer le processus pédagogique, pourtant il était évident que le nœud du problème était ailleurs.

8. Voir aussi le commentaire qu'en fait S. BEAUD dans le numéro du 5/11 mars 2003 des Inrockuptibles.

9. Je ne parle pas de châtiments corporels, pratiquement éliminés aujourd'hui, mais des divers processus de « bridage».

10. Certainement le Nanouk de R. FLAHERTY, M. GOYET mentionne notamment la construction de l'igloo, dôme protecteur de glace dont la symbolique pourrait être interrogée.

11. Il rappelle aussi que le recrutement des enseignants se fait de plus en plus dans des milieux socio-culturellement «éloignés» de la population scolaire « de base», en tout cas de celle chez qui la «Crise de l'éducation» est la plus patente.

12. Le mythe grec, bien sûr, n'a pas été écrit pour les lecteurs du XXIe siècle et je m'intéresse à ce qu'il suscite chez nous et non à un illusoire sens "prophétique»: à simple titre d'exemple, la façon dont le concept de sacré / divin se répartit dans les deux ordres mental / humain et corporel / animal était dans la Grèce archaïque bien différente de la nôtre.

13. «Celui qui contraint vigoureusement à reculer loin», mais l'idée de «loin» me paraît recouvrir une fonction plus évacuatrice qu'initiatrice et le reste de l'interprétation est discutable. 14. Et je me range à l'opinion d'Y. CHEVALLARD qui réclame une véritable réflexion sur une déontologie professionnelle de l'enseignement.

\section{RÉSUMÉS}

Dans le cadre d'un module de formation IUFM «Corps et Voix", l'auteur a été amené à s'interroger sur l'image de l'enseignant véhiculée par l'iconographie historique et contemporaine. La recherche des critères visuels fait émerger un geste / signe dont 
l'interprétation conduit à l'idée d'un corps enseignant qui semble posé comme transparent ; ce corps ainsi évacué serait-il un corps redouté ? On peut le penser si l'on songe aux problèmes de violence scolaire largement médiatisés. Le récent ouvrage de M. GOYET témoigne du malaise éprouvé par nombre d'enseignants. Mais une réflexion appuyée sur l'utilisation pédagogique que fait S. BOISMARE du mythe d'Héraclès et Eurysthée conduit à repenser le problème et à s'interroger sur les rapports du corps à la norme et sur les modalités de formation des futurs enseignants.

In the context of a IUFM « Body and Voice » formation unit, the author has been led to wonder what was the teacher's image worn by historic and contemporary iconography. Searching visual standards raised up a gesture / sign which suggested a teaching body defined as transparent; could this evacuated body be a dreaded body? This supposition is allowed considering wide broadcast matters of school violence. M. GOYET'S recent book testify discomfort felt by many teachers. However, a reflection based on the S. BOIMARE'S pedagogic use of Hercules and Eurysthee myth drives to reconsider the problem and wonder about the relation between body and norm and the training of future teachers.

\section{INDEX}

Mots-clés : conflit, corps, formation des maîtres, image de l'enseignant

Keywords : body, conflict, teacher image, teacher training

\section{AUTEUR}

\section{GÉRARD SAEZ}

IUFM de Montpellier 